\title{
Congenital Diaphragmatic Hernia: Antenatal Diagnosis and Successful Repair In Preterm Neonate Case Report
}

\author{
Wasmi AL - FADHLI ${ }^{1}$, Sulaiman AL-MUNAIFI ${ }^{2}$, Ibrahim A. ABDELAZIM³ \\ Kuwait
}

\begin{abstract}
Most of the congenital diaphragmatic hernias observed in the left hemi diaphragm. The pathogenesis of congenital diaphragmatic hernias is not completely understood; but abnormal development of the diaphragm at 6-10 weeks' supposed to be the cause in most of the cases. Although congenital diaphragmatic hernias usually occurs sporadically, environmental exposures have been implicated. Improvement of the antenatal imaging techniques has allowed early diagnosis and evaluation of the associated anomalies. In antenatally diagnosed congenital diaphragmatic hernias cases, multidisciplinary team management and delivery at tertiary centers with proper facilities may be provided to optimize the outcome.
\end{abstract}

A 39-year-old woman, with the history of one previous cesarean section, was admitted to the hospital at 25 weeks' gestation, because of placenta previa and her antenatal ultrasound showed fetal congenital diaphragmatic hernias with polyhydramnios. During hospitalization, she was monitored for hemoglobin levels, consumptive coagulopathy, and fetal well-being. Betamethasone was given to accelerate the fetal lung maturity, and magnesium sulphate for fetal neuro-protection. Due to an attack of heavy antepartum hemorrhage, delivery occurred at the gestational age of 28 weeks +6 days. The delivered female newborn was admitted to the neonatal intensive care unit because of respiratory distress and prematurity, and received surfactant, dobutamine and intravenous antibiotics. Chest examination of the studied neonate showed diminished air entry on the left side, and chest X-ray showed stomach shadow in the left hemi thorax. After exclusion of congenital heart diseases and intracranial hemorrhage, the baby was successfully operated on the postpartum $7^{\text {th }}$ day, and discharged from the neonatal intensive care unit 60 days after the operation.

Two months after discharge from the neonatal intensive care unit, examination of the studied neonate was successfully operated on the postpartum $7^{\text {th }}$ day showed normal growth parameters and appropriate motor and sensory development for her age.

Antenatal diagnosis of congenital diaphragmatic hernias allows multidisciplinary team management and delivery at tertiary center with the proper facilities to optimize the outcome.

Keywords: Congenital diaphragmatic hernia, Prenatal diagnosis, Repair, Premature

\section{Gynecol Obstet Reprod Med 2018;24(2):104-107}

${ }^{1}$ Department of Neonatal Surgery Ahmadi Hospital Kuwait Oil Company (KOC), Kuwait.

${ }_{2}^{2}$ Department of Neonatology Ahmadi Hospital Kuwait Oil Company (KOC), Kuwait.

${ }^{3}$ Department of Obstetrics, and Gynecology Ahmadi Hospital Kuwait Oil Company (KOC), Kuwait.

Address of Correspondence: Ibrahim A. Abdelazim Consultant of Obstetrics, and

Gynecology, Ahmadi Hospital, Kuwait

Oil Company (KOC), Kuwait.

dr.ibrahimanwar@gmail.com

Submitted for Publication:

01.11 .2017

Accepted for Publication:

19.11.2017

\begin{tabular}{|c|c|}
\hline & Access this article online \\
\hline $\begin{array}{c}\text { Quick Response Code: } \\
\text { and }\end{array}$ & $\begin{array}{r}\text { Website: www.gorm.com.tr } \\
\text { e-mail: info@gorm.com.tr }\end{array}$ \\
\cline { 2 - 3 } & DOI:10.201613/GORM.2017.746 \\
\hline
\end{tabular}

How to cite this article: Fadhl WA. Munaifi SA. Ibrahim A. Abdelazim IA. Congenital Diaphragmatic Hernia: Antenatal Diagnosis and Successful Repair In Preterm Neonate Case Report. Gynecol Obstet Reprod Med 2018;24(2):104-107

\section{Introduction}

Congenital diaphragmatic hernia $(\mathrm{CDH})$ is a rare disorder which involves the left hemi diaphragm in $75 \%$ of the cases; $15 \%$ are right-sided and $10 \%$ bilateral (1). The pathogenesis is not completely understood; but abnormal development of the diaphragm at 6-10 weeks' gestation supposed to be the cause in most of the cases. Although most cases occur sporadically, environmental exposures have been implicated, including smoking, alcohol, vitamin A deficiency, thalidomide, and anticonvulsants (2-5).

With the improvement of the antenatal imaging techniques, early diagnosis of $\mathrm{CDH}$ has been possible as well as the evaluation of the associated anomalies and syndromes. Associated anomalies, most commonly of renal, gastrointestinal, cardiovascular and central nervous systems, found in $40 \%-60 \%$ of live-born infants with $\mathrm{CDH}(6,7)$.

Antenatal diagnosis of $\mathrm{CDH}$ allows multidisciplinary team management involving an obstetrician, neonatologist, and pe- 
diatric surgeon, and delivery at tertiary center with the proper facilities to optimize the outcome $(8,9)$.

This report represents a case of $\mathrm{CDH}$ diagnosed antenatally, to highlight the importance of early diagnosis, and the good outcome when managed through multidisciplinary team approach in a tertiary center.

\section{Case Report}

A 39-year-old woman, with the history of one previous cesarean section, was admitted to the hospital at 25 weeks' gestation, after a mild attack of antepartum hemorrhage due to placenta previa. Her antenatal ultrasound showed fetal CDH (Bochdalek hernia) and polyhydramnios, but no other associated anomalies. During hospitalization, she was monitored for hemoglobin levels, consumptive coagulopathy via platelet count, prothrombin, bleeding and clotting times, and fetal well-being and informed consent was obtained. Hemoglobin measurements as well as tests for consumptive coagulopathy were performed especially during active bleeding. Fetal wellbeing was evaluated by fetal movement counts, daily fetal heart rate (FHR) recordings, and weekly trans-abdominal ultrasonographic examinations to measure amniotic fluid volume, fetal growth and umbilical artery Doppler indices $(10,11)$. Betamethasone was given to accelerate the fetal lung maturity, and magnesium sulphate for fetal neuro-protection the day after admission $(12,13)$.

Due to an attack of heavy antepartum hemorrhage, delivery occurred at the gestational age of 28 weeks +6 days. The delivered female newborn was 960 grams, with APGAR scores of 5, 6, and 8, at 1, 5 and 10 minutes respectively. She was admitted to the neonatal intensive care unit (NICU) because of respiratory distress and prematurity, placed on continuous mandatory ventilation (CMV) and received surfactant after the delivery, and monitored by oxygen saturation and blood gases. Intravenous (IV) fluids given were monitored by the input/output fluid chart. Additionally, she received dobutamine, and IV antibiotics according to the results of the cultures performed as a part of neonatal sepsis screening protocol.

On admission to NICU, chest examination of the studied neonate has revealed diminished air entry on the left side, with displacement of the heart to the right side; and chest X-ray has shown the stomach shadow in the left hemi thorax, pushing the heart to the right side of the chest (Figure 1).

Echocardiographic examination performed two days after NICU admission, revealed a small atrial septal defect (ASD) and patent ductus arteriosus (PDA), which were considered normal for a premature baby, and the cranial ultrasound showed no intracranial hemorrhage (ICH).

The studied neonate was successfully operated on the postpartum 7th day when her cardio-pulmonary functions were stable, and the diaphragmatic defect identified (Figure 2) was repaired after reduction of the contents to the abdominal cavity using interrupted stitches $(9,14)$. (Figure 3).

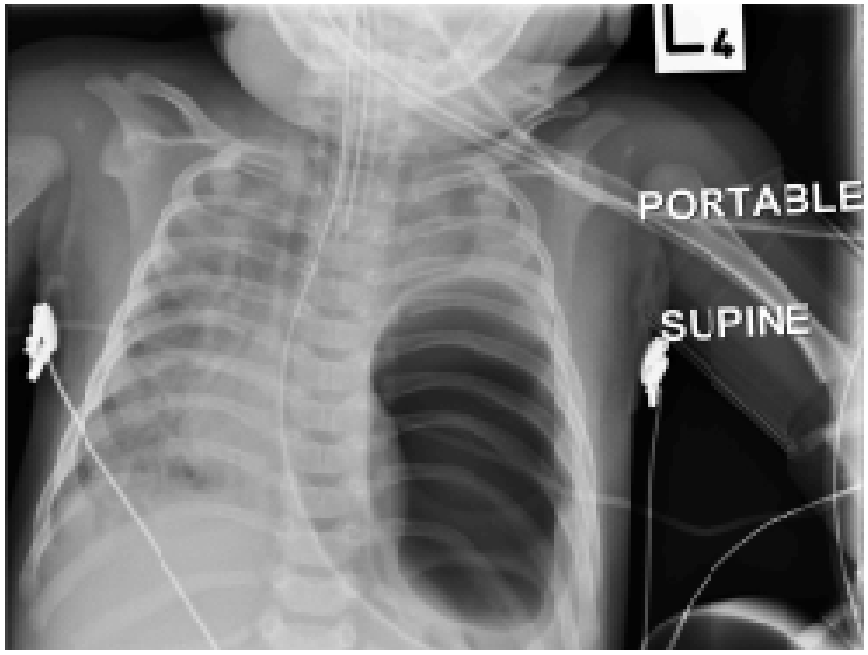

Figure 1: Chest $X$ ray of the studied neonate before surgery showing the stomach in the chest, pushing the heart to the right side.

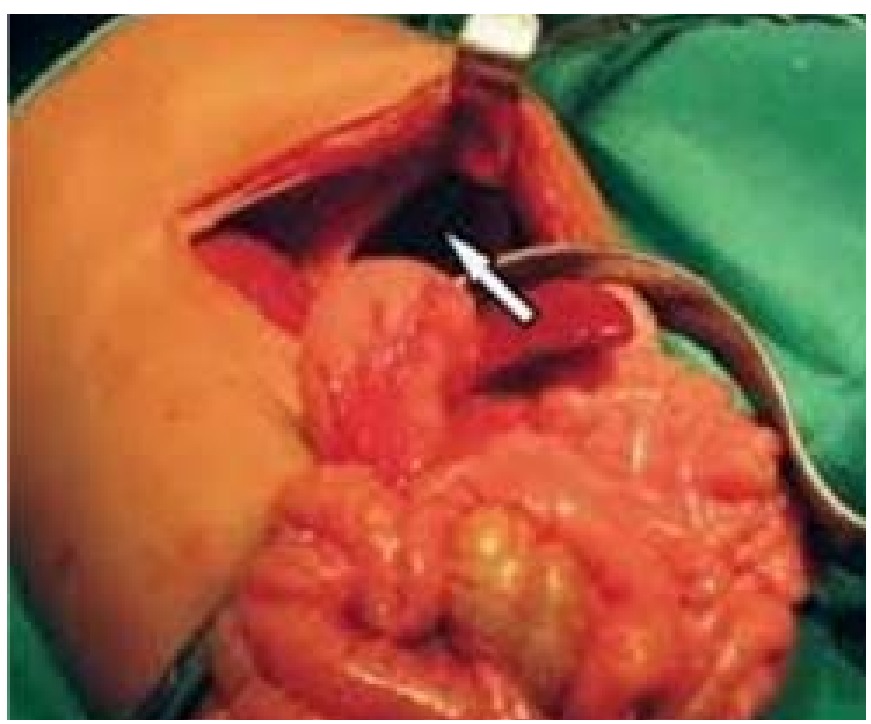

Figure 2: Intra-operative image shows the congenital diaphragmatic hernia on the left side after reduction of its contents

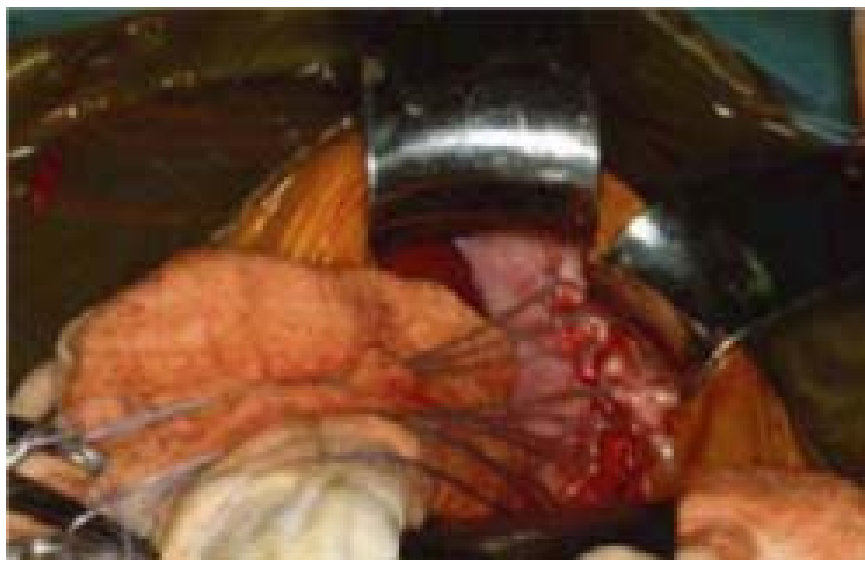

Figure 3: Intra-operative image shows the repaired congenital diaphragmatic hernia on the left side, using interrupted stitches 
On the postoperative $3^{\text {rd }}$ day, the studied neonate shifted to synchronized intermittent-mandatory ventilation (SIMV), and dobutamine infusion was completely stopped. On the $12^{\text {th }}$ day, she shifted to pressure support ventilation (PSV), oral feeding started through orogastric tube, and the rates of IV fluids and total parenteral nutrition (TPN) were reduced. On the $21^{\text {st }}$ day, she was extubated and placed on nasal continuous positive airway pressure (CPAP).

Forty days after the operation, her weight increased to 1421 grams, oral feeding rate was increased, IV fluids and TPN were stopped, and nasal CPAP was shifted to heating, ventilation and air conditioning (HVNC).

The small ASD and PDA were closed spontaneously, and she was 2120 grams and on complete oral feeding and room air when discharged from the NICU 60 days after the operation for out-patient follow-up (Figure 4).

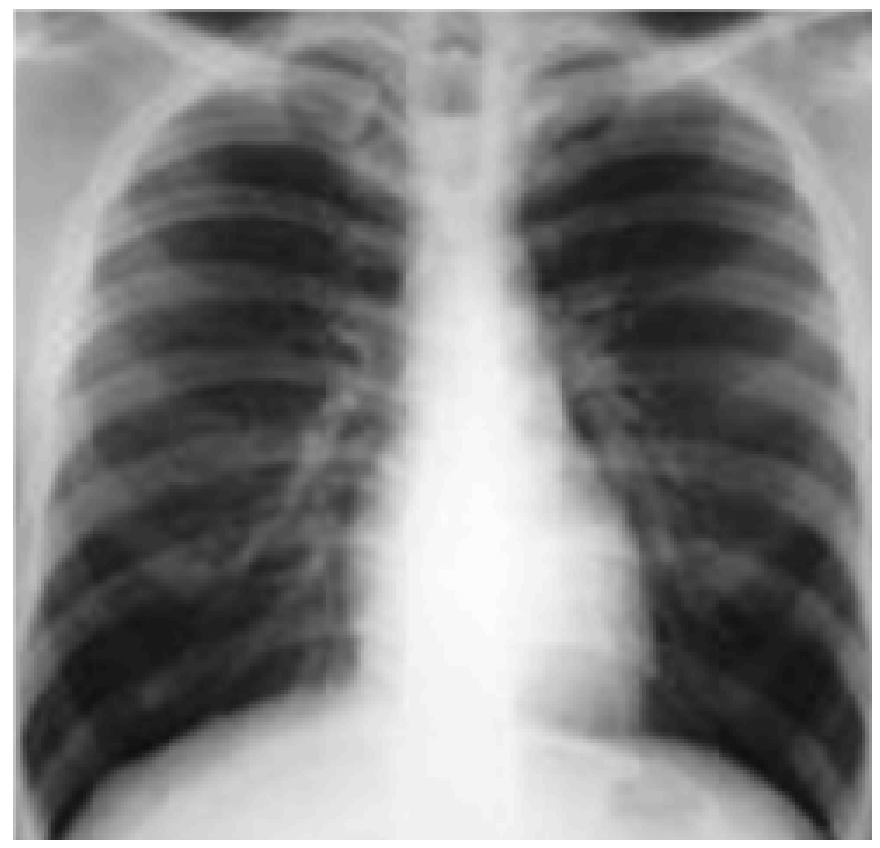

Figure 4: Chest X-ray of studied neonate before discharge from the hospital

Two months after discharge from the NICU, examination of the studied neonate at the out-patient department showed normal growth parameters and appropriate motor and sensory development for her age.

This case report was approved by the hospital institute ethical committee and informed consent was taken from the parents of the baby.

\section{Discussion}

The incidence of the $\mathrm{CDH}$ ranges between 1:2000 and 1:5000 live births (15). The mortality rate in neonates with $\mathrm{CDH}$ remains high with an overall survival rate of around $60 \%(15,16)$.
Congenital diaphragmatic hernia is one of the major challenges of perinatal medicine and surgery, and antenatal diagnosis allows multidisciplinary team management to optimize the outcome (9).

Infants with $\mathrm{CDH}$ usually present with respiratory distress due to pulmonary hypoplasia, which require endotracheal intubation, and pressor support (17).

Surgical management of the $\mathrm{CDH}$ involves reduction of the herniated abdominal content, and repair of the diaphragmatic defect. Researchers agree in that, repair of the diaphragmatic defect should be delayed until the cardio-pulmonary functions of the newborn are stable $(9,14)$.

Traditionally, the repair performed via an open surgical approach, using either a patch repair or a muscle flap; the minimally invasive techniques may be associated with a higher rate of hernia recurrence (18). In the current case report, $\mathrm{CDH}$ was diagnosed antenatally at 25 weeks' gestation and was managed by a multidisciplinary team. Delivery was planned in a tertiary center with intensive care facilities. Immediately after the delivery, the studied neonate was admitted to the NICU because of respiratory distress, prematurity and low birthweight, as the gestational age was 28 weeks +6 days at delivery and the birthweight 960 grams; then operated successfully on the $7^{\text {th }}$ postpartum day, after stabilization of cardio-pulmonary functions. She was discharged from the NICU 60 days after the operation when she was 2120 grams, and two months later out-patient examination showed normal growth parameters and appropriate motor and sensory development for her age.

Tsao et al, have emphasized that the outcomes in preterm infants with $\mathrm{CDH}$ are worse compared to term infants, the survival rate being around $50 \%$ in preterm infants after surgical correction (19).

A systematic review of controlled trials showed that the neonatal survival improved in infants diagnosed prenatally and delivered at a tertiary center with intensive care facility (20).

Mortality was higher in the presence of additional anomalies, iatrogenic lung injury, pulmonary hypoplasia, and pulmonary hypertension $(19,20)$.

Tovar, has notified that, the best hospital reported survival rate was $80 \%$ in $\mathrm{CDH}$, but it remained around $50 \%$ in population-based studies (9).

Taking the current case into consideration, it may be concluded that, antenatal diagnosis of $\mathrm{CDH}$ allows multidisciplinary team approach and delivery at tertiary center with proper facilities to optimize the outcome.

Antenatal diagnosis of CDH allows multidisciplinary team approach and delivery at tertiary center with proper facilities to optimize the outcome. 
: Ethical approval: The case report approved by the institute ethical committee of Ahmadi hospital and informed consent taken from the studied woman.

Acknowledgement: Authors are grateful to the studied woman, agreed to participate in this presentation.

Disclosure: The presentation funded by the authors themselves.

Conflict of interest: No conflict of interest related to this presentation.

\section{References}

1. Zaiss I, Kehl S, Link K, Neff W, Schaible T, Sütterlin M, et al. Associated malformations in congenital diaphragmatic hernia. Am J Perinatol 2011;28(3):211-8.

2. Caspers KM, Oltean C, Romitti PA, Sun L, Pober BR, Rasmussen SA, et al. National Birth Defects Prevention Study. Maternal periconceptional exposure to cigarette smoking and alcohol consumption and congenital diaphragmatic hernia. Birth Defects Res A Clin Mol Teratol 2010;88(12):1040-9.

3. Felix JF, de Jong EM, Torfs CP, de Klein A, Rottier RJ, Tibboel D. Genetic and environmental factors in the etiology of esophageal atresia and/or tracheoesophageal fistula: an overview of the current concepts. Birth Defects Res A Clin Mol Teratol 2009 Sep;85(9):747-54.

4. Yang W, Shaw GM, Carmichael SL, Rasmussen SA, Waller DK, Pober BR, et al. National Birth Defects Prevention Study. Nutrient intakes in women and congenital diaphragmatic hernia in their offspring. Birth Defects Res A Clin Mol Teratol 2008;82(3):131-8.

5. Enns GM, Cox VA, Goldstein RB, Gibbs DL, Harrison MR, Golabi M. Congenital diaphragmatic defects and associated syndromes, malformations, and chromosome anomalies: a retrospective study of 60 patients and literature review. Am J Med Genet 1998;79:215-225. 1998/10

6. Skari H, Bjornland K, Haugen G, Egeland T, Emblem R. Congenital diaphragmatic hernia: a meta-analysis of mortality factors. J Pediatr Surg 2000;35(8):1187-97.

7. Brownlee EM, Howatson AG, Davis CF, Sabharwal AJ. The hidden mortality of congenital diaphragmatic hernia: a 20-year review. J Pediatr Surg 2009 Feb;44(2):317-20.

8. Mohammed Al Ghafri, Ibtisam Al Sidairi, Madhavan Nayar. Late Presentation of Congenital Diaphragmatic Hernia: A Case Report. Oman Med J 2014;29(3):232-3.

9. Tovar JA. Congenital diaphragmatic hernia. Orphanet J
Rare Dis 2012 Jan 3;7:1.

10. Frenckner BP, Lally PA, Hintz SR, Lally KP; Congenital Diaphragmatic Hernia Study Group. Prenatal diagnosis of congenital diaphragmatic hernia: how should the babies be delivered? J Pediatr Surg 2007;42(9):1533-8.

11. Safavi A, Lin Y, Skarsgard ED; Canadian Pediatric Surgery Network. Perinatal management of congenital diaphragmatic hernia: when and how should babies be delivered? Results from the Canadian Pediatric Surgery Network. J Pediatr Surg 2010;45(12):2334-9.

12. Danzer E, Hedrick HL. Neurodevelopmental and neurofunctional outcomes in children with congenital diaphragmatic hernia. Early Hum Dev 2011;87(9):625-32.

13. Danzer E, Gerdes M, Bernbaum J, D1Agostino J, Bebbington M, Siegle J, et al. Neurodevelopmental outcome of infants with congenital diaphragmatic hernia prospectively enrolled in an interdisciplinary follow-up program. J Pediatr Surg 2010;45(9):1759-66.

14. Moyer V, Moya F, Tibboel R, Losty P, Nagaya M, Lally KP. Late versus early surgical correction for congenital diaphragmatic hernia in newborn infants. Cochrane Database Syst Rev 2002;(3):CD001695.

15. Abdul Haium AA, Sim SW, Ong LY, Rajadurai VS. Congenital diaphragmatic hernia associated with oesophageal atresia and tracheo-oesophageal fistula in a low birth weight infant. BMJ Case Rep 2013 Aug 20;2013. pii: bcr2013200014.

16. The Congenital Diaphragmatic Hernia Study Group Estimating disease severity of congenital diaphragmatic hernia in the first 5 minutes of life. J Pediatr Surg 2001; 2013:141-5

17. Mugford M, Elbourne D, Field D. Extracorporeal membrane oxygenation for severe respiratory failure in newborn infants. Cochrane Database Syst Rev 2008;(3): CD001340.

18. Tsao K, Lally PA, Lally KP; Congenital Diaphragmatic Hernia Study Group. Minimally invasive repair of congenital diaphragmatic hernia. J Pediatr Surg 2011;46(6): 1158-64.

19. Tsao K, Allison ND, Harting MT, Lally PA, Lally KP. Congenital diaphragmatic hernia in the preterm infant. Surgery 2010 Aug;148(2):404-10.

20. Logan JW, Rice HE, Goldberg RN, Cotten CM. Congenital diaphragmatic hernia: a systematic review and summary of best-evidence practice strategies. J Perinatol 2007;27(9):535-49. 